\title{
Atitude e comportamento de consumo em um destino ecológico: Um estudo com jovens de Ilhabela (SP, Brasil)
}

\author{
Attitude and behavior of consumption in an ecological destination: \\ A study with youths of Ilhabela (SP, Brazil)
}

\section{La actitud y el comportamiento del consumo en un destino ecológico: Un estudio con jóvenes de Ilhabela (SP, Brasil)}

\author{
Braulio Oliveira ${ }^{1}$ \\ Suzane Strehlau ${ }^{2}$ \\ Régis Thomaz Ximenes ${ }^{3}$
}

\begin{abstract}
Resumo: A preservação do meio ambiente vem sendo discutida há bastante tempo na mídia e no meio acadêmico. $O$ objetivo deste trabalho foi verificar a atitude e o comportamento de jovens de uma estância ecoturística, quanto ao consumo ecologicamente correto. A pesquisa foi realizada com uma amostra probabilística de 143 estudantes de ensino médio de Ilhabela, cidade do litoral norte do Estado de São Paulo, que tem como uma das principais atividades econômicas o turismo ecológico. As análises realizadas apontam que a população pesquisada pode ser dividida em três grupos em relação à atitude e ao comportamento de consumo ecologicamente correto, os quais foram rotulados de "proativo", de "indiferente" e de "negativo"; mostram, ainda, que 10 das 40 assertivas da escala utilizada para mensurar o fenômeno discriminam os grupos e geram uma função que permite a alocação preditiva adequada de $92,3 \%$ da população pesquisada. A maioria dos perfis considerados "proativos", gerados a partir da combinação das variáveis "gênero", "ano escolar que cursa" e "renda", é formado por mulheres. Conclui-se que é imperativo trabalhar a questão da preservação ambiental junto aos jovens, pois apenas $21 \%$ da população foram considerados "proativos".
\end{abstract}

Palavras-chave: Comportamento do consumidor; Destino ecológico; Meio ambiente; Sustentabilidade.

Abstract: The preservation of the environment has been discussed for a long time in the media and in the academy. The aim of this study was to exam attitudes and behavior of young people from an ecotourism destination regarding environmental consumption. The survey was conducted with a random sample of 143 high school students from Ilhabela, a city on the northern coast of São Paulo, which the main economic activity is ecotourism. The analyses indicate that the studied population can be divided into three groups relating the attitude and behavior of environmentally friendly consumption. These groups were labeled "proactive". "Indifferent" and "negative", 10 of the 40 statements of the scale used to measure the phenomenon discriminate the groups and generate a function that allows predictive adequate allocation of

\footnotetext{
${ }^{1}$ Doutor em Administração pela FEA/USP. Professor Pesquisador do PPGA-FEI. E-mail: braulio@fei.edu.br

${ }^{2}$ Doutora em Administração pela EAESP/FGV, Professora Pesquisadora do PPGA-Uninove. E-mail: strehlau@gmail.com

${ }^{3}$ Mestre em Administração pela FEl. Professor da Faculdade Drummond. E-mail: regisximenes@gmail.com
} 
92.3\% of the population. Most profiles considered "proactive" were generated from the combination of variables "gender", "current school year" and "income" and is comprised of women. We conclude that it is imperative to address the issue of environmental preservation among young people, because only $21 \%$ of the population was considered "proactive".

Keywords: Consumer behavior; Ecological destination; Environment; Sustainability.

Resumen: La preservación del medio ambiente se ha discutido durante mucho tiempo en las media y en la academia. El objetivo de este estudio fue examinar las actitudes y comportamientos de los jóvenes de un destino de ecoturismo en relación al consumo ecológicamente correcto. La investigación se realizó con una muestra aleatoria de 143 estudiantes de enseno medio de Ilhabela, una ciudad en la costa norte de São Paulo, en que la principal actividad económica es el ecoturismo. Los análisis indican que la población estudiada se puede dividir en tres grupos que se refieren a la actitud y el comportamiento de consumo. Estos grupos fueron nombrados de "proactivo", "indiferente" y "negativo"; 10 de las 40 afirmaciones de la escala utilizada discriminan los grupos y generó una función que permitió la clasificación predictiva adecuada de 92,3\% de la población. La mayoría de los perfiles considerados "proactivo" se generaron a partir de la combinación de las variables "género", "año escolar en curso" y "renta" y está compuesta por mujeres. Llegamos a la conclusión de que es indispensable abordar el tema de la preservación del medio ambiente entre los jóvenes, ya que sólo el $21 \%$ de la población se considera "activo".

Palabras clave: Comportamiento del consumidor; Destino ecológico; Medio ambiente; Sostenibilidad.

\section{INTRODUÇÃO}

A sustentabilidade ambiental tem sido objeto de discussão nos meios empresarial, governamental e acadêmico. Fundamentalmente, ela está relacionada com a capacidade de uma sociedade suprir as necessidades da geração presente sem afetar a competência das gerações futuras de garantir as suas próprias (Heilbroner, 1980).

No que tange ao consumo, um dos grandes catalizadores da degradação ambiental, Kotler (2006) ressalta a necessidade de que ele deve levar em conta a preservação ambiental, caso contrário, gerações futuras poderão herdar um ônus econômico e social intolerável, resultante do esgotamento de recursos e da poluição decorrente do uso indiscriminado de produtos que deterioram o meio ambiente. Inclusive, para determinados setores de atividade econômica, como é o caso do turismo, em especial, o turismo ecológico, a preservação do meio ambiente é crucial.

Portanto, especificamente no que diz respeito ao ecoturismo, é imperativa a necessidade de que tanto turistas quanto as populações das localidades que o exploram possuam atitudes e comportamentos compatíveis com a sobrevivência do destino no longo prazo (Herbig \& O'hara, 1997), o que passa pelo consumo.

No entanto, pesquisas que abordam o ecoturismo tem se focado principalmente nas atitudes e comportamentos do turista (Tangeland, 2011; Naidoo, Ramseook-Munhurrun \& Seegoolam, 2011), ou em aspectos da administração (Fredman \& Tyrväinen, 2010; Huang \& Confer, 2009; Rønningen, 2010). A população local ainda recebe pouca atenção, embora seja reconhecida a sua importância no processo de desenvolvimento (Byrd, 2007).

Em vista do exposto, este trabalho teve por objetivo conhecer as atitudes e 
comportamentos relacionados ao consumo ambientalmente adequado de uma população residente em uma localidade que tem o seu desenvolvimento econômico vinculado ao turismo ecológico. Para tanto, além do referencial teórico pertinente, foi realizada uma pesquisa de campo com jovens da cidade de Ilhabela, conforme será detalhada mais adiante.

\section{FUNDAMENTAÇÃO TEÓRICA}

Em uma análise exaustiva Fennel (2007) diferenciou turismo de natureza do ecoturismo. $\mathrm{O}$ turismo baseado na natureza engloba outras formas de turismo como de massa, de aventura, de baixo impacto e ecoturismo que usam recursos da natureza na sua forma selvagem ou sem desenvolvimento (Fennel, 2007); já o ecoturismo é sustentável - uma forma não invasiva de turismo, baseado no aprendizado sobre a natureza, que é eticamente gerenciada para ter baixo impacto, com orientação local, e deve contribuir para a conservação da área (Fennel, 2007).

Assim, o turismo ecológico requer que os diversos públicos interagentes (stakeholders) estejam envolvidos no processo do desenvolvimento do turismo sustentável (Byrd, 2007; Byrd \& Gustke, 2011). Mas apesar disso, Jöhr (1994) afirma ser pequena a parcela da população que efetivamente adota um comportamento ambientalmente favorável. Por outro lado, pesquisa do Instituto Akatu (2002) aponta que os jovens são mais dispostos a conhecer novas experiências e são mais abertos às mudanças e introdução de novos hábitos e mentalidade.

No que tange ao consumo, Vermeir e Verbeke (2006) afirmam haver falta de coerência entre as preocupações expressas pelos consumidores e seus comportamentos quanto às escolhas, pois eles estão muitas vezes "aprisionados" a padrões de consumo insustentáveis. Isso significa que eles estão ligados em restrições econômicas, barreiras institucionais ou desigualdades no acesso a serviços que incentivam ativamente comportamentos insustentáveis (Yates, 2008).

No Brasil, pesquisa realizada pelo Ibope mostra que embora $85 \%$ dos entrevistados para avaliar as empresas com boas práticas sociais estariam dispostos a pagar mais por um produto feito sem prejudicar o ambiente, apenas $37 \%$ pararia de comprar um produto se soubessem que sua marca favorita de que a empresa seria prejudicial para o ambiente (Palhano, 2007).

Alguns estudos vêm apontando haver relação entre determinadas características dos indivíduos e o consumo ecologicamente correto. Belz e Peattie (2009), por exemplo, apontam haver correlação positiva com a renda; e, Steger e Meima (1998) apontam haver correlação positiva com a idade. No primeiro, a lógica está em privilegiar aspectos de diferenciação e que podem conferir algum status, uma vez que são produtos, normalmente, mais caros que os seus semelhantes que não denotam preocupação ambientalmente correta; no segundo, o fato de que à medida que a idade aumenta, a experiência e preocupações mais relacionadas à qualidade de vida vão ganhando espaço. Daí depreende-se, também, que a escolaridade pode possuir importância significativa, uma vez que em certo grau pode ser associado à idade e de outra forma ao conhecimento acerca de questões gerais da sociedade.

Além disso, pode-se acreditar que o contexto no qual o indivíduo está inserido afeta o seu 
grau de consciência acerca da relevância de algo. Assim, é de se supor que um indivíduo que vive em um contexto em que o desenvolvimento de sua comunidade dependa da preservação ambiental, sua propensão ao consumo ambientalmente favorável seja alta.

\section{Escala de mensuração}

Uma escala que vem sendo utilizada para se mensurar atitudes e comportamentos de consumo ambientalmente adequado foi desenvolvida por Antil e Bennett em 1979, intitulada "Comportamento de Consumo Socialmente Responsável". Trata-se de um instrumento composto de 40 assertivas que abordam situações relacionadas a consumo e a meio-ambiente, sobre as quais os respondentes assinalam o seu grau de concordância em uma escala Likert de cinco pontos, conforme apresentada no Anexo A. O instrumento é apontado como confiável e de fácil aplicabilidade (Squire, Juric \& Cornwell, 2001).

Ressalte-se que mais recentemente vem-se discutindo e concluindo haver diferenças entre atitude e comportamento, e que, esse último, trata-se basicamente da efetivação, da ação (Mowen \& Minor, 2003; Motta \& Rossi, 2003; Oliveira \& Gouvêa, 2010). Assim, por se tratar de um instrumento de coleta que solicita aos pesquisados que atribuam ordenamento às assertivas nele contidas, entendeu-se que sua finalidade é de mensurar diretamente a atitude e, indiretamente, o comportamento, já que ela é apontada como um forte influenciador do comportamento (Mowen \& Minor, 2003; Motta \& Rossi, 2003; Oliveira \& Gouvêa, 2010).

\section{METODOLOGIA DA PESQUISA DE CAMPO}

A população da pesquisa foi a de estudantes de ensino médio de escolas públicas de Ilhabela. A cidade fica em uma ilha localizada no litoral norte do Estado de São Paulo. Possuía uma população residente de 28.176 em 2010, e uma área $348 \mathrm{~km}^{2}$ (IBGE, 2010). Trata-se de uma estância ecoturística, que possui um dos cenários mais belos de toda costa brasileira, reunindo praias espremidas entre o Oceano Atlântico e as montanhas da Serra do Mar. Em setembro de 1934 foi emancipada definitivamente do Município de São Sebastião recebendo o atual nome em 1944. Situa-se a 190 Km de São Paulo mais 15 minutos de balsa, a partir de São Sebastião.

Ilhabela possui oito escolas de ensino médio, sendo cinco públicas e três particulares. No ano de 2007 foram matriculados nas escolas públicas 1.013 alunos e 106 alunos fizeram suas matrículas nas escolas privadas de ensino médio.

Julgou-se o ambiente escolar apropriado, uma vez que se pôde pesquisar uma grande quantidade de jovens em um curto espaço de tempo, otimizando os recursos envolvidos na pesquisa. A escolha por escolas públicas deveu-se ao fato de que a maior parte desses estudantes frequentam os estabelecimentos públicos de ensino (IBGE, 2010) e, além disso, deve-se supor que as instituições públicas de ensino cumprem a Constituição Federal do Brasil (1988), que estabelece responsabilidade ao poder público em "promover a educação ambiental em todos os níveis do 
ensino e a conscientização pública para a preservação do meio ambiente" (art. 225, §1으, VI/CF) e a Lei 9.795 (1999), que versa sobre a educação ambiental, afirmando que ela deve estar presente em todos os níveis e modalidades do processo educativo. Também a cidade escolhida obedeceu ao objetivo definido no trabalho, uma vez que se trata de um local cujo desenvolvimento econômico está vinculado ao turismo ecológico.

A amostragem foi probabilística aleatória simples, tendo sido realizada em duas etapas: na primeira, foi sorteada uma escola entre as públicas; na segunda etapa, foram sorteados os alunos da escola selecionada, a partir de listas fornecidas por ela própria.

Das cinco escolas públicas de ensino médio de Ilhabela, a sorteada foi a E.E. Professor Gabriel ribeiro dos Santos. Foram pesquisados 150 jovens dos quais 143 formulários foram considerados válidos, produzindo um erro amostral também aproximado de $8 \%$ para um nível de significância de $95 \%$. Os questionários, de auto-resposta, foram distribuídos pelos pesquisadores aos alunos sorteados, com a permissão da diretora da escola.

O instrumento de coleta de dados era composto de duas partes: a primeira possuía perguntas sobre o perfil demográfico do respondente e, a segunda, o questionário de Antil e Bennet (1979), o qual pode ser observado no Anexo A.

Os questionários foram recolhidos, tabulados e analisados pelos próprios pesquisadores, com o software SPSS versão 17.

Para se alcançar o objetivo proposto neste trabalho, além da análise descritiva, foram utilizadas as seguintes técnicas inferenciais (Hair et al., 2006):

- Análise de Clusters: para se verificar como os respondentes podem ser agrupados;

- Análise Discriminante: para se verificar quais variáveis são mais representativas em discriminar os respondentes e se gerar um modelo preditivo de alocação;

- Análise de Correspondência (Anacor): para se verificar a aderência dos agrupamentos de respondentes com as variáveis de perfil (demográficas).

Em razão da grande quantidade de variáveis no questionário utilizado, foi iniciado o processo de análise fatorial, com o objetivo de agrupar as variáveis em fatores. $O$ teste de Bartlett tem associado um nível de significância inferior a 0,01 ( $p$-valor $<0,01)$, o que leva à rejeição da hipótese de que a matriz de correlações seja igual à matriz de identidade, mostrando, portanto, que existe correlação entre as variáveis, o que, por sua vez, indica a pertinência do uso do modelo fatorial. Entretanto, o KMO igual a 0,718 indica a existência de coeficientes de correlação parciais pequenos. De fato, analisando-se a matriz de correlações, a maior correlação existente é de 0,529, o que se dá entre as variáveis 21 e 27. Desta forma, optou-se pelo não agrupamento das variáveis, ou seja, pela não continuidade da análise fatorial, a fim de se evitar perder informações importantes para as demais análises.

Foi realizado o teste de Kolmogorov-Smirnov para verificar a normalidade de cada uma das variáveis. Os resultados indicaram que não se pode rejeitar $\mathrm{HO}$, de que cada uma das variáveis possui distribuição normal ao nível de significância de 0,01 ( $p$-valor<0,01). 


\section{ANÁLISE DOS RESULTADOS}

A análise de clusters foi realizada pelo método between groups e com medida pela distância quadrática euclidiana. A partir da análise dos coeficientes, os dados apontam como adequada a formação de três clusters. O cluster 1 é formado por 30 casos, o cluster 2 por 91 casos, o cluster 3 por 22 casos. Com base na média geral de pontuação obtida na escala utilizada, os clusters foram rotulados da seguinte forma:

- Cluster 1, "atitude proativa";

- Cluster 2, "Atitude indiferente";

- Cluster 3, "atitude negativa".

A fim de se observar se as diferenças entre os grupos são significativas estatisticamente e quais variáveis possuem maior poder discriminatório, procedeu-se a Análise Discriminante. Foi utilizado o método stepwise e o critério Wilks' Lambda, tendo sido consideradas iguais as probabilidades a priori de alocação dos elementos nos grupos.

Os resultados mostram que com exceção das variáveis v3, v8, v15, v18, v25, v28 e v29, todas as demais discriminam os três clusters formados. Mostram, ainda, que a variável v31 foi a primeira a ser incluída no modelo gerado, pois é a que possui maior poder discriminatório, apresentando um valor de Wilks' Lambda de 0,674 e um valor de F de 33,819. Por sua vez, a última a ser incluída no modelo gerado foi a variável v15, que apresenta um valor de Wilks' Lambda de 0,674 e um valor de $\mathrm{F}$ de 33,819.

A correlação canônica, que mede a associação entre os escores discriminantes é próxima de 1 nas duas funções geradas, o que se considera adequado para a qualidade do modelo, conforme observa-se na Tabela 1 (Hair et al., 2006). Observa-se, ainda, que a primeira função gerada explica $75 \%$ da variância e a segunda, $25 \%$.

Tabela 1. Qualidade do modelo

\begin{tabular}{rrrrr}
\hline Função & Eigenvalue & Variância (\%) & Variância Acumulada (\%) & Correlação Canônica \\
\hline 1 & 2,9 & 75,0 & 75,0 &, 865 \\
2 &, 98 & 25,0 & 100,0 &, 705 \\
\hline
\end{tabular}

Fonte: Dados coletados processados.

Como o método stepwise prioriza as variáveis com maior poder discriminatório e inclui apenas as mais representativas, as variáveis efetivamente consideradas são as apresentadas no Quadro 1. 
Quadro 1. Assertivas incluídas no modelo discriminante

4. A poluição é, atualmente, um dos problemas mais críticos de nosso país.

9. A poluição não está particularmente afetando minha vida.*

10. Os consumidores devem ser obrigados a pagar preços mais elevados para os produtos que poluem o meio ambiente.

11. Eu realmente fico indignado em pensar que o governo não está fazendo mais para ajudar a controlar a poluição do meio ambiente.

12. Garrafas, latas ou outros produtos que não possam ser reciclados, deveriam ser proibidas por lei sua devolução ao meio ambiente.

15. O Governo Federal deve subsidiar pesquisas sobre tecnologias de reciclagem de produtos e resíduos. 16. Eu estou disposto a ir para o trabalho de bicicleta ou a pé, a fim de reduzir a poluição do ar.

19. Esta questão da poluição nunca me preocupou, pois sinto que há muito exagero nela.*

21. Eu estaria disposto a ter minha roupa menos branca ou macia em troca da certeza de que estaria usando um produto não poluente na sua lavagem.

22. Os fabricantes devem ser obrigados a utilizar materiais reciclados na fabricação de produtos e nos processos operacionais.

23. Acho que as pessoas deveriam conversar com seus amigos para não utilizarem produtos que poluam ou prejudiquem o ambiente.

24.Propagandas e comerciais devem ser obrigados a mencionar as desvantagens ecológicas dos produtos.

31. Eu estaria disposto a pagar mais um "real" a cada mês para a produção de eletricidade, se significar um ar mais limpo.

35. As pessoas devem estar dispostas a aceitar fumaça (poluição) em troca da comodidade dos automóveis. 36. Quando penso nas formas que algumas indústrias poluem, fico frustrado e indignado.

40. Eu raramente me preocupo com os efeitos da fumaça (poluição) sobre mim e minha família. *

* Assertivas que possuem escala invertida. Fonte: Dados coletados processados.

De acordo com os resultados de classificação a partir da melhor função gerada (a primeira, pois apresentou o Wilk's Lambda de 0,127 , o mais próximo de 0), 92,3\% dos casos foram alocados corretamente, o que se pode considerar um excelente índice.

Para a realização da análise de correspondência as variáveis de gênero, ano escolar que cursa e renda foram testadas isoladamente e de forma combinada. O melhor resultado foi obtido com a combinação das três variáveis, a qual foi denominada "perfil", de acordo com a apresentação no Quadro 2. 
Quadro 2. Características de cada perfil gerado

\begin{tabular}{|c|c|}
\hline Perfil & Características \\
\hline 1 & Homem classe $C$, do $1^{\circ}$. ano \\
\hline 2 & Homem classe $B$, do $1^{\circ}$. ano \\
\hline 3 & Homem classe $A$, do $1^{\circ}$. ano \\
\hline 4 & Homem classe $\mathrm{C}$, do $2^{\circ}$. ano \\
\hline 5 & Homem classe $B$, do $2^{\circ}$. ano \\
\hline 6 & Homem classe $A$, do $2^{\circ}$. ano \\
\hline 7 & Homem classe $\mathrm{C}$, do $3^{\circ}$. ano \\
\hline 8 & Homem classe $B$, do $3^{\circ}$. ano \\
\hline 9 & Homem classe $A$, do $3^{\circ}$. ano \\
\hline 10 & Mulher classe $C$, do $1^{\circ}$. ano \\
\hline 11 & Mulher classe $B$, do $1^{\circ}$. ano \\
\hline 12 & Mulher classe $A$, do $1^{\circ}$. ano \\
\hline 13 & Mulher classe $\mathrm{C}$, do $2^{\circ}$. ano \\
\hline 14 & Mulher classe $\mathrm{B}$, do $2^{\circ}$. ano \\
\hline 15 & Mulher classe $A$, do $2^{\circ}$. ano \\
\hline 16 & Mulher classe $\mathrm{C}$, do $3^{\circ}$. ano \\
\hline 17 & Mulher classe $B$, do $3^{\circ}$. ano \\
\hline 18 & Mulher classe $A$, do $3^{\circ}$. ano \\
\hline
\end{tabular}

Fonte: Dados coletados processados.

Assim, a análise de correspondência mostrou-se adequada, uma vez que o teste Quiquadrado $\left(X^{2}\right)$ associado resultou um $p$-valor $=0,047$, o que demonstra haver relação de dependência entre a variável considerada (perfil) e os clusters, ao nível de significância de 0,05, conforme se apresenta na Tabela 2.

Tabela 2. Nível de significância do relacionamento entre a variável perfil e os clusters

\begin{tabular}{crrrr}
\hline Teste & Valor do Teste & Graus de Liberdade & \multicolumn{1}{c}{$\boldsymbol{p}$ valor } \\
\hline Pearson Chi-Square & 48,947 & 34 &, 047 \\
\hline
\end{tabular}

Fonte: Dados coletados processados.

As dimensões 1 e 2 do mapa gerado explicam 66,6\% e 33,4\% da inércia total, o que indica que a dimensão 1 é a mais importante para a interpretação dos resultados. A correlação entre os valores próprios (eigenvalues) ou singulares de ambas as dimensões é pequena $(0,016)$, 
garantindo a localização dos pontos na dimensão correta. A variância dos valores próprios em ambas as dimensões são muito pequenas $(0,071)^{2}$ e $(0,063)^{2}$, respectivamente, garantindo que a Anacor é estável, ou seja, produzirá a mesma solução para uma amostra semelhante da mesma população.

Em relação à variável em linha "perfil", o que mais contribui para a inércia da dimensão 1 é a 14 (mulheres de alta renda que cursam o 2‥ ano), com 0,531. Já a variável em coluna "cluster", a que mais contribui é a "negativo", com 0,849.

O mapa gerado com cruzamento das dos perfis com os agrupamentos indica que os perfis $1,10,11,15,17$ e 18 (homem classe $C$ do $1^{\circ}$ ano, mulher classe $C$ do $1^{\circ}$ ano, mulher classe $B$ do $1^{\circ}$ ano, mulher classe $A$ do $2^{\circ}$ ano, mulher classe $B$ do $3^{\circ}$ ano, e mulher classe $A$ do $3^{\circ}$ ano) são mais aderentes ao grupo "proativo", que os perfis $2,4,6,8,9,12$ e 13 (homem classe $B$ do $1^{\circ}$ ano, homem classe $C$ do $2^{\circ}$ ano, homem classe $A$ do $2^{\circ}$ ano, homem classe $B$ do $3^{\circ}$ ano, homem classe $A$ do $3^{\circ}$ ano, mulher classe $A$ do $1^{\circ}$ ano, e mulher classe $C$ do $2^{\circ}$ ano) são mais aderentes ao grupo "indiferente", e que os perfis 3 e 14 (homem classe A do $1^{\circ}$ ano, e mulher classe B do $2^{\circ}$ ano) são mais aderentes ao grupo "negativo".

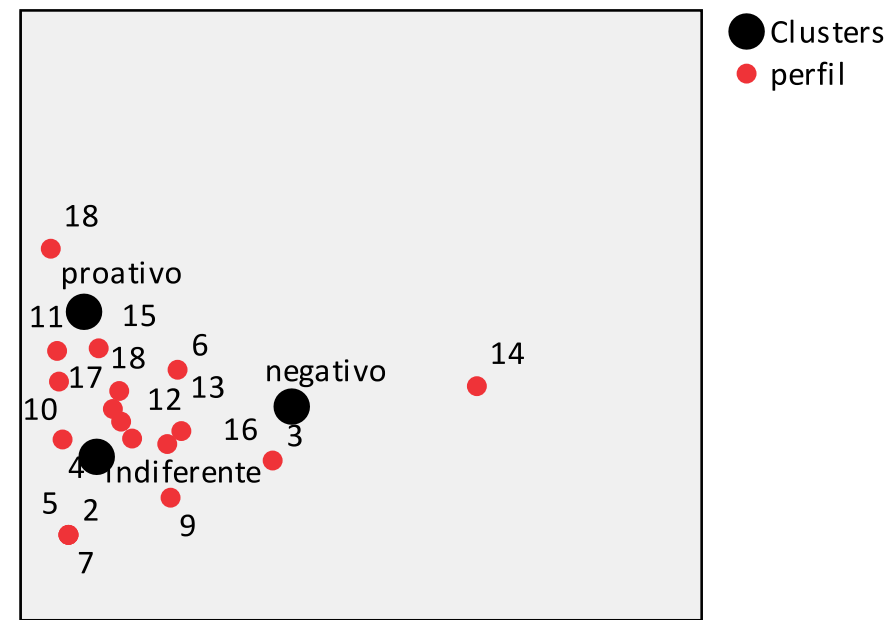

Figura 1. Anacor entre agrupamentos e perfis Fonte: Dados coletados processados.

\section{CONSIDERAÇÕES FINAIS}

Embora tenha sido encontrado um grupo, que possui atitude e comportamento proativos em relação ao meio ambiente, ele representa apenas $21 \%$ da população pesquisada, o que se pode considerar muito pouco, haja vista toda a informação disponível na mídia com respeito ao consumo e à sustentabilidade ambiental, especialmente pelo fato de serem estudantes e moradores de uma cidade que depende economicamente do turismo ecológico. Por outro lado, deve-se ressaltar que apenas $15 \%$ da população possuem atitude e comportamento negativo em relação ao meio ambiente. 
A análise discriminante, baseada na escala utilizada apontou as assertivas que contribuem para que os respondentes possam ser incluídos nos grupos "proativo", "indiferente" e "negativo", indicando caminhos para se melhorar a atitude e o comportamento da população em relação à questão.

Já a análise de correspondência permitiu enxergar a aderência de cada um dos perfis criados a partir do cruzamento das variáveis de gênero, ano que está cursando no ensino médio e renda em relação a cada um dos agrupamentos. Nota-se que dos seis perfis aderentes ao grupo "proativo" apenas um é do gênero masculino, e que dos sete perfis aderentes ao grupo "indiferente" apenas dois são do gênero feminino. Assim, embora o gênero não tenha sido significante estatisticamente para discriminar os grupos, julga-se importante que estudos adicionais sejam feitos para se verificar especificamente esta questão.

Por fim, com base no referencial teórico e nos resultados da pesquisa de campo, julga-se fundamental que se trabalhe com extremo empenho os jovens, no sentido de que sejam reais defensores do meio ambiente. E certamente a escola, um dos locais onde passam boa parte desta etapa da vida, é um importante local para que essa questão seja trabalhada, somadas às iniciativas do governo, de organizações da sociedade civil e até mesmo de empresas, tanto em suas ações, como em suas exposições na mídia.

Sugere-se que futuros estudos sobre o assunto abordado neste trabalho considerem outras localidades geográficas e perfis populacionais.

\section{REFERÊNCIAS}

Antil, J. H., \& Bennett, P. D. (1979). Construction and validation of a scale to measure socially responsible consumption behavior. In K. E. Henion, \& T. C. Kinnear (Eds.). The Conserver Society (pp. 51-68). Chicago: American Marketing Association.

Belz, F.-M., \& Peattie, K. (2009). Sustatinability marketing. A global Perspective. Chichester: John Wiley \& Sons.

Byrd, E. T. (2007). Stakeholders in sustainable tourism and their role: applying stakeholder theory to sustainable development. Tourism Review, 62(2), 6-13.

Byrd, E. T.; Gustke, L. (2011). Using decision trees to identify tourism stakeholders. Journal of Place Management and Development, 4(2), 148-168.

Chan, R.Y.K., \& Lau, L. L. B. Y. (2000). Antecedents of green purchases: a survey in china. The Journal of Consumer Marketing, 17(4), 338-357.

Constituição Federal do Brasil de 1988 (2001). [Coleção Saraiva de Legislação]. São Paulo: Saraiva.

Fennel, D. A. (2007). Ecotourism. London: Routledge.

Fredman, P., \& Tyrväinen, L. (2010). Frontiers in nature-based tourism. Scandinavian Journal of Hospitality \& Tourism, 10(3), 177-189.

Hair, J. F. Jr. et al. (2006). Análise multivariada de dados. Porto Alegre: Bookman.

Hall, C., \& Boyd, S. (2005). Nature-based tourism in peripheral areas: development or disaster? Clevedon: 


\section{Cromwell Press.}

Heilbroner, R. L. (1987). A formação da Sociedade Econômica. Rio de Janeiro: Guanabara.

Herbig, P., \& O'hara, B. (1997). Ecotourism: a guide for marketers. European Business Review, 97(5).

Huang, C., \& Confer, J. (2009). Applying the Tourism Opportunity Spectrum model in nature-based tourism management. Managing Leisure, 14(4), 247-257.

IBGE (2010). Recuperado em 25 janeiro 2012, de http://www.ibge.gov.br/cidadesat/topwindow.htm?1.

Instituto Akatu (2002). Os jovens e o consumo sustentável: construindo o próprio futuro? São Paulo: Akatu.

Jöhr, H. (1994). O verde é negócio. São Paulo: Saraiva.

Kotler, P. (2006). Administração de marketing. São Paulo: Pearson.

Lei n. 9.795, de 27 de abril de 1999 (1999). Dispõe sobre a educação ambiental, institui a Política Nacional de Educação Ambiental e dá outras providências. Diário Oficial da União. Brasília, DF: Presidência da República - Casa Civil - Subchefia para Assuntos Jurídicos.

Motta, S. L. S., \& Rossi, G. B. (2003). A influência do fator ecológico na decisão de compra de bens de conveniência: um estudo exploratório na cidade de São Paulo. Revista de Administração, 38(1), 46-57.

Mowen, J. C., \& Minor, M. S. (2003). Comportamento do consumidor. São Paulo: Prentice Hall.

Naidoo, P., Ramseook-Munhurrun, P., \& Seegoolam, P. (2011). An assessment of visitor satisfaction with nature-based tourism attractions. International Journal of Management \& Marketing Research, 4(1), 8798.

Oliveira, B., \& Gouvêa, M. A. (2010). A importância das ações sociais empresariais nas decisões de compra dos consumidores. Gestão \& Produção, 17(4), 791-800.

Palhano, A. (2007). Consumidor não usa critério socioambiental. Folha de São Paulo, 27 nov. 2007, p. B11.

Rønningen, M. (2010). Innovative processes in a nature-based tourism case: the role of a tour-operator as the driver of innovation. Scandinavian Journal of Hospitality \& Tourism, 10(3), 190-206.

Steger, U., \& Meima, R. (1998). The strategic dimensions of environmental management: sustanining the corporation during the age of ecologicla discovery. London: Macmillan.

Tangeland, T. (2011). Why do people purchase nature-based tourism activity products? a norwegian case study of outdoor recreation. Scandinavian Journal of Hospitality \& Tourism, 11(4), 435-456.

Vermeir, I., \& Verbeke, W. (2006). Sustainable consuption: exploring the consumer attitude-behavioral intention gap. Journal of Agricultural and Environmental Ethics, 19(2), 169-194.

Yates, L. (2008). Sustainable consumption: the consumer perspective. Consumer Policy Review, 18(4), 9699.

Artigo recebido em: 31/05/2012.

Artigo aprovado em: 29/08/2012. 